\title{
Association of age and macular pigment optical density using dual-wavelength autofluorescence imaging
}

This article was published in the following Dove Press journal:

Clinical Ophthalmology

3 April 2013

Number of times this article has been viewed

\author{
Verônica Castro Lima ${ }^{1,2}$ \\ Richard B Rosen ${ }^{1,3}$ \\ Tiago Santos Prata ${ }^{2}$ \\ Syril Dorairaj ${ }^{4}$ \\ Leigh Spielberg' \\ Mauricio Maia ${ }^{2}$ \\ Juliana M Sallum²
}

'Retina Service, Department of Ophthalmology, The New York Eye and Ear Infirmary, New York, NY, ${ }^{2}$ Department of Ophthalmology, Federal University of São Paulo, São Paulo, Brazil; ${ }^{3}$ New York Medical College, New York, NY, ${ }^{4}$ Department of Ophthalmology, Mayo Clinic, Jacksonville, FL, USA
Correspondence: Richard B Rosen Retina Service, Department of Ophthalmology, The New York Eye and Ear Infirmary, 310 East 14th Street, New York, NY, USA 10003

$\mathrm{Tel}+\mid 2129794181$

Fax + I 2129794268

Email rrosen@nyee.edu
Background: Several lines of evidence suggest that macular pigment may play a protective role against age-related macular degeneration, but the influence of age on macular pigment density levels remains unclear. This study was designed to investigate the relationship between age and the normal distribution of macular pigment optical density (MPOD) values surrounding the fovea.

Methods: Consecutive healthy subjects with no evidence of ocular disease were enrolled in this study. After inclusion, MPOD values were measured at specific eccentricities $(0.5,1$, and 2 degrees) from the foveal center using a dual-wavelength autofluorescence method employing a modified confocal scanning laser ophthalmoscope. Whenever both eyes were eligible, one was randomly selected for analysis. The correlation between age and MPOD values was investigated using regression analysis.

Results: Thirty subjects (30 eyes) were included (mean age $48.6 \pm 16.4$ [range 23-77] years). Significant differences were found between MPOD values measured at $0.5,1$, and 2 degrees from the center of the fovea $(0.49 \pm 0.12$ density units, $0.37 \pm 0.11$ density units, and $0.13 \pm 0.05$ density units, respectively, $P<0.05)$. Significant correlations between age and MPOD values at 0.5 and 1 degree were found $(P \leq 0.02)$. Values measured at 2 degrees did not correlate significantly with age $(P=0.06)$.

Conclusion: In healthy subjects, MPOD values were highest near the foveal center. These values appeared to increase during adulthood (peak at 45-50 years), followed by a gradual reduction after 60 years of age.

Keywords: age, macular pigment optical density, dual-wavelength autofluorescence imaging, association

\section{Introduction}

Macular pigment consists of two major dietary carotenoids, lutein and zeaxanthin, which act as an optical filter for wavelengths shorter than $550 \mathrm{~nm}$ and provide antioxidant protection to the human retina by inhibiting the peroxidation of long-chain polyunsaturated fatty acids. ${ }^{1-3}$ The highest levels of macular pigment in the human body are measured at the Henle fiber layer in the fovea, which consists of the axons of the foveal cones, and in the inner plexiform layer at the parafoveal area. ${ }^{46}$ The concentration of macular pigment peaks in the center of the fovea (foveola), and decreases 100-fold within a few millimeters of eccentricity. Lutein is more prevalent in the peripheral retina, given that the ratio of lutein to zeaxanthin changes from approximately $1: 2.4$ in the central retina to $2: 1$ in the peripheral retina. ${ }^{7} \mathrm{~A}$ third macular carotenoid called mesozeaxanthin appears to be derived from retinal lutein and is the 
predominant $\mathrm{Z}$ isomer at the foveal center. ${ }^{89}$ Because oxidative damage seems to be an important factor for exacerbation of several retinal diseases, ${ }^{10-12}$ the postulated protective role of macular pigment in some disorders, such as age-related macular degeneration (AMD) and diabetic retinopathy, has been investigated. ${ }^{3,13-15}$

Following this rationale, a study has shown that three major risk factors for AMD, ie, increasing age, family history, and cigarette smoking, were significantly associated with a relative lack of macular pigment, and dietary and serum lutein were positively correlated with macular pigment. ${ }^{14}$ However, the relationship between the spatial profile of macular pigment and risk factors for this disease has not been established.

The spatial distribution of macular pigment around the fovea appears to vary among normal individuals. ${ }^{16-19}$ Variation with eccentricity of the lutein to zeaxanthin ratio has been suggested to correspond to the rod to cone ratio, possibly due to preferential accumulation in these structures. However, immunohistochemical localization of lutein and zeaxanthin binding proteins in the primate retina has shown equal staining of both rods and cones. ${ }^{20}$ The importance of such variation in spatial distribution (such as the presence of a central dip in some individuals) is not yet elucidated, but may be related to a protective role of these carotenoids against some retinal diseases, such as AMD. ${ }^{19}$

Several techniques have been used to measure macular pigment density indirectly and noninvasively in vivo. They are categorized as either psychophysical or optical methods, and each one has merits and limitations. The dual wavelength autofluorescence method, one of the more recently introduced methods, provides objective and reliable measurements of macular pigment distribution and is well suited to clinical testing of patients. ${ }^{19,21,22}$

Given that macular pigment has been proposed as a protective factor against AMD and age is a major risk factor for this disease, a fundamental question to be answered is whether there is any age association for macular pigment in normal healthy subjects. This study was designed to survey such a group of normal subjects and compare the distribution of MPOD with their age distribution.

\section{Materials and methods}

This cross-sectional, comparative, noninterventional protocol was conducted at the Department of Ophthalmology of The New York Eye and Ear Infirmary after approval by its institutional review board, and adhered to the tenets of the Declaration of Helsinki. Informed consent was obtained from all subjects prior to enrolment and examination.

\section{Subjects}

Consecutive eligible healthy volunteers were enrolled for this study. They were evaluated in the comprehensive Ophthalmology Clinic, and those who met our inclusion criteria were directed to the Retina Clinic, where a complete ophthalmological examination was performed, including funduscopy with dilated pupils. Exclusion criteria included spherical equivalent outside the range of $\pm 4 \mathrm{D}$, presence of any ocular diseases or media opacities (any degree of cataract), previous intraocular surgery or ocular trauma, current consumption of oral carotenoids, presence of diabetes, and best-corrected visual acuity worse than 20/20. If both eyes were eligible, one eye was randomly selected. Demographic data collected included age, gender, and ethnicity. Lifestyle and general health data were also obtained.

\section{Procedures}

Dual wavelength autofluorescence imaging for measuring MPOD is an objective method that has been described elsewhere. ${ }^{19-26}$ Briefly, it relies on the autofluorescence of lipofuscin, which is located in the retinal pigment epithelial cells. This fluorescence is emitted in the $520-800 \mathrm{~nm}$ spectral range, and can be excited in vivo using light between $400 \mathrm{~nm}$ and $570 \mathrm{~nm} .{ }^{27}$ In the fovea, because the excitation spectrum of foveal lipofuscin closely matches the absorption spectrum of macular pigment, light absorbed by carotenoids results in a central zone of reduced lipofuscin fluorescence. To measure the MPOD, the autofluorescence method compares results from the region of maximal macular pigment density overlying the fovea with that of an area with no optically appreciable macular pigment several degrees eccentric to the fovea, using two excitation wavelengths that are differentially absorbed by the macular pigment $(488 \mathrm{~nm}$ is well absorbed and $512 \mathrm{~nm}$ is minimally absorbed).

The imaging device used to perform the test is a specially modified Heidelberg Retinal Angiography Scanning Laser Ophthalmoscope (SLO) (HRA1, Heidelberg Engineering Inc, Heidelberg, Germany). All pupils were dilated with tropicamide $1 \%$ and phenylephrine $2.5 \%$ drops prior to testing, and each eye was measured three times. Subjects were positioned in front of the scanning laser ophthalmoscope and instructed to maintain steady fixation straight ahead. Once the operator was comfortable with the stability of the subject's fixation and the quality of the macular image, rapid sequences of 20 degree fields were captured at $488 \mathrm{~nm}$ and $514 \mathrm{~nm}$. Macular pigment optical density maps were generated by the proprietary system software which digitally subtracted the log autofluorescence images, and calculated 
mean MPOD values for 0.5 and 2 degree diameter circles centered on the fovea. The reference eccentricity from the center of the fovea, where MPOD was assumed to be zero, was set at 7.0 degrees. For each eye tested, the average of two good quality maps was used for analysis. Criteria for good quality images included sharp focus, absence of artifacts, and accurate centration within the posterior pole. Poor quality images were not considered for analysis. Values for MPOD are reported in dimensionless density units (DU).

\section{Statistical analysis and sample size calculation}

Values of MPOD were compared between each retinal eccentricity using analysis of variance. The StudentNewman-Keuls test was used for all pairwise comparisons. The association between age and MPOD values in each region surrounding the fovea was evaluated using regression analysis. Whenever both eyes were eligible, one eye was randomly selected for analysis. Statistical significance was set at $P<0.05$ and computerized analysis was performed using MedCalc software (MedCalc Inc, Mariakerke, Belgium).

Considering normal MPOD values (at 0.5 degrees) as approximately 0.50 density units (DU) based on previously published data ${ }^{15,22}$ and a statistical power of $80 \%$ (beta, 0.20 ; alpha, 0.05), it would be necessary to test $25 \mathrm{sub}$ jects to detect a mean difference between eccentricities of
0.05 DU (10\% difference, estimated standard deviation of differences approximately $0.08 \mathrm{DU})$.

\section{Results}

In this study, 30 normal subjects (30 eyes) were included. The mean age of the patients was $48.6 \pm 16.4$ (23-77) years. The majority of patients were female $(70 \%)$ and Caucasian (76.7\%). Significant differences were found for MPOD values measured at $0.5,1$, and 2 degrees from the center of the fovea $(0.49 \pm 0.12 \mathrm{DU}, 0.37 \pm 0.11 \mathrm{DU}$, and $0.13 \pm 0.05 \mathrm{DU}$, respectively, $P<0.05$, Figure 1).

Significant correlations between age and MPOD values at 0.5 degree $\left(\mathrm{R}^{2}=0.32, P=0.006\right)$ and 1 degree $\left(\mathrm{R}^{2}=0.34\right.$, $P=0.016)$ were found. Values measured at 2 degrees did not correlate significantly with age $\left(\mathrm{R}^{2}=0.15, P=0.06\right)$. As shown in Figure 2, we found nonlinear correlations between age and MPOD values in different eccentricities. Based on these scatter diagrams (distribution of MPOD values according to age), we estimated that higher MPOD values were distributed between 45 and 55 years of age (especially at 0.5 and 1 degree from the center of the fovea). MPOD levels tended to increase in adulthood, followed by a gradual reduction that continued with aging (Figure 2).

\section{Discussion}

This study was designed to investigate the distribution of MPOD surrounding the fovea at specific retinal locations

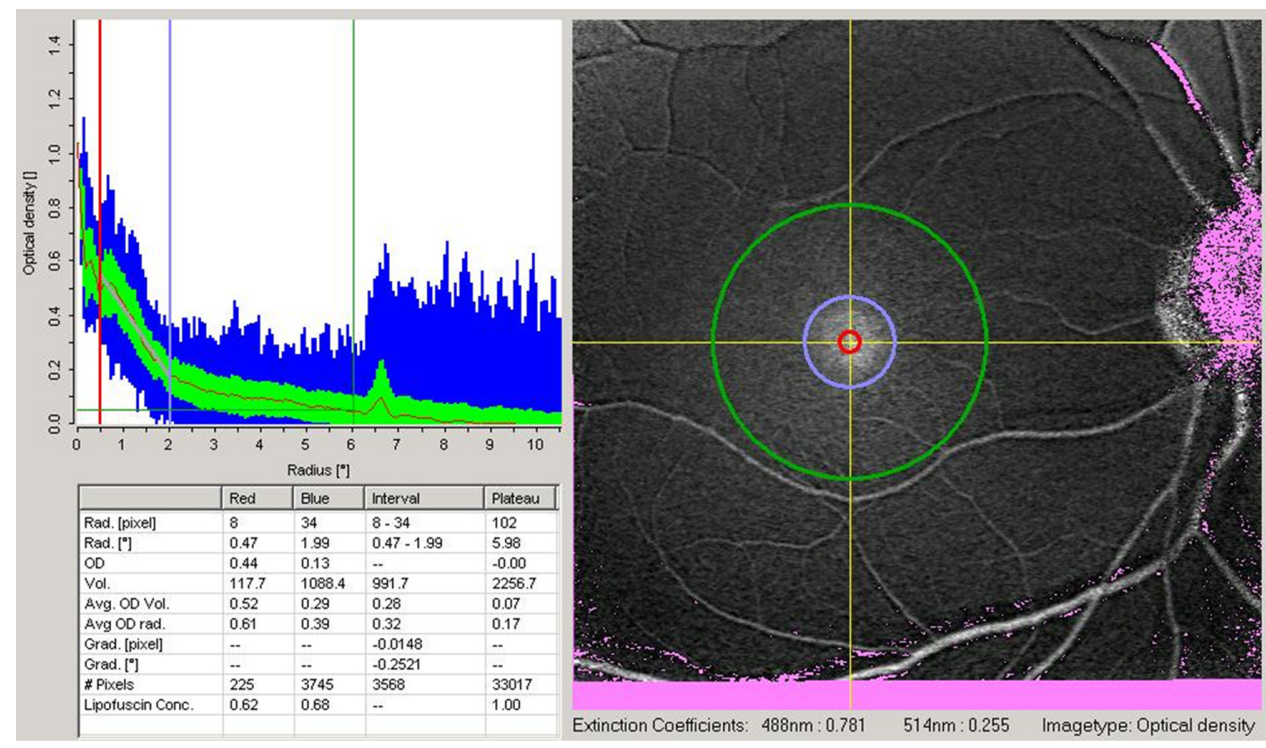

Figure I Macular pigment optical density map of one eye included in the study.

Notes: The image analyzed is demonstrated on the right side of the dialog. Two yellow lines mark the point used for radial analysis. The red and blue circles demarcate areas of 0.5 and 2.0 degrees from the center of the fovea, respectively. On the upper left side of the dialog there is a diagram with a normal radial distribution of macular pigment optical density around the center of the fovea. On the lower left side, the box shows numerical values for macular pigment optical density in the specific eccentricities analyzed. 

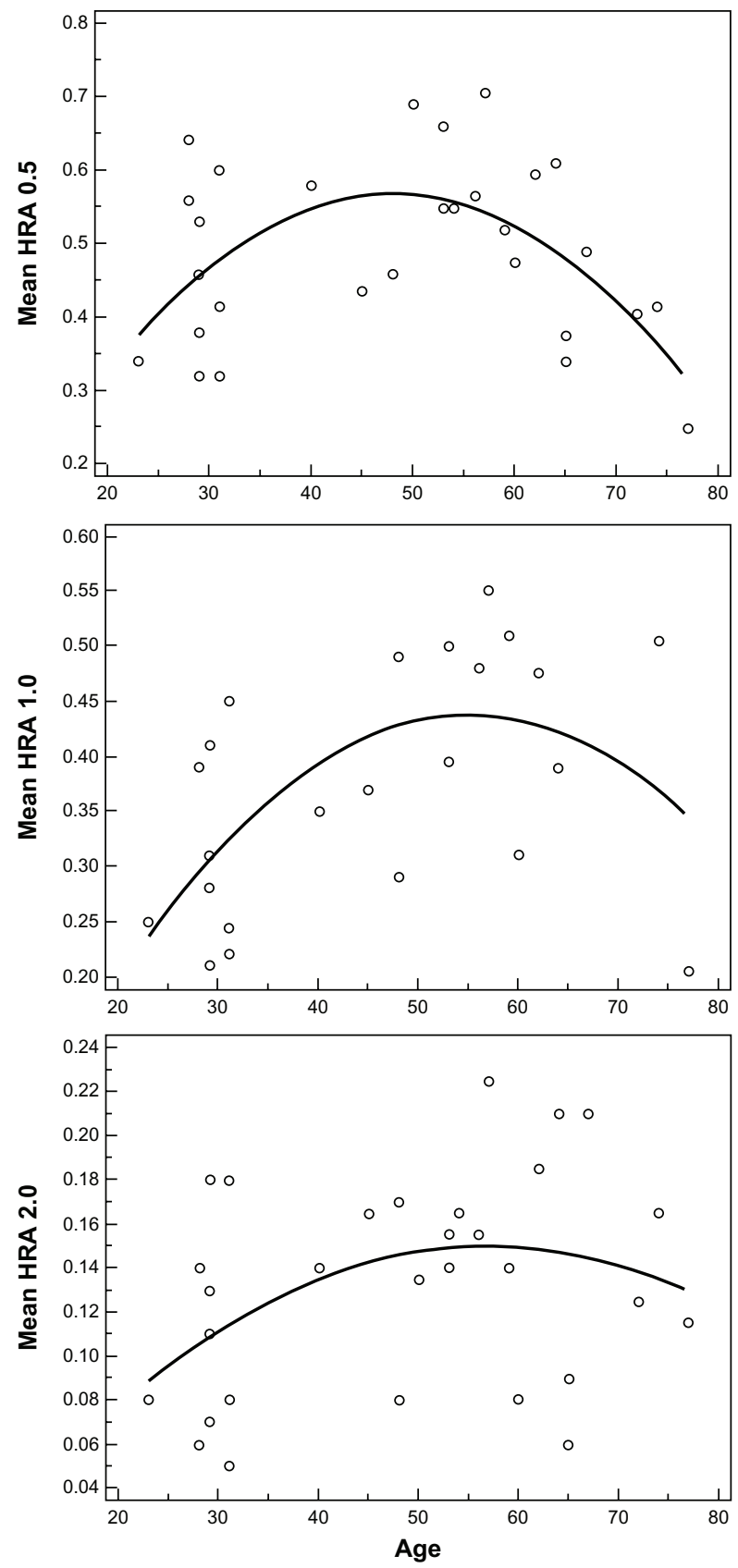

Figure 2 Macular pigment optical density values at $0.5,1.0$, and 2.0 degrees of retinal eccentricity versus age.

Abbreviation: HRA, Heidelberg Retinal Angiography.

and the association of age with these values using the dualwavelength autofluorescence method described previously by Lima et al ${ }^{15}$ and Canovas et al. ${ }^{22}$ Our findings confirm that levels of MPOD decrease consistently with eccentricity from the center of the fovea in a normal population, which is consistent with the body of literature developed using heterochromatic flicker photometry. ${ }^{28}$ Interestingly, following peaking for values in subjects in their mid 50s, MPOD levels show a significant decline with age.
The retina contains highly unsaturated lipids susceptible to oxidative damage in a region of high oxygen tension and light exposure. Oxidative stress is considered an important mechanism for the pathogenesis of AMD, and antioxidant nutrients have been shown to play a role in protection against the disease. ${ }^{13,29}$ There is growing evidence suggesting a relationship between levels of macular pigment and risk of age-related eye diseases, although a direct link has not yet been established. Additionally, known risk factors for AMD such as cigarette smoking, light-colored irides, age, obesity, and female gender have been associated with low levels of MPOD. ${ }^{14,30}$ Age is an established risk factor for AMD, so an understanding of macular pigment levels in healthy subjects may be revealing.

The association between aging and MPOD has been previously addressed in the literature, but remains controversial, with conflicting results, largely because of the methodologies used or population inconsistencies. Some studies have shown no age dependence for MPOD, ${ }^{31-33}$ while others have demonstrated a decline $e^{14,30,34-36}$ or an increase in MPOD values with age. ${ }^{37,38}$ When analyzing these findings, it is important to consider two factors, ie, the technique used to measure MPOD and the age range of the subjects studied. Some studies using heterochromatic flicker photometry showed no association between MPOD and age, but failed to account for confounding variables related to MPOD. ${ }^{31,33}$ Berendschot and van Norren ${ }^{32}$ were unable to demonstrate any association between MPOD and age in a normal population with a wide age range using five different methods for MPOD measurement, including the autofluorescence technique. One previous study ${ }^{37}$ found a significant and positive effect of age on MPOD in 435 elderly subjects with and without AMD. Similarly, Dietzel et a ${ }^{38}$ demonstrated a slight increase in MPOD with age in an elderly population with a limited age range, in part using supplements containing lutein and/ or zeaxanthin, and the majority had some degree of AMD.

A number of studies have shown an inverse association between age and MPOD. Nolan et $\mathrm{al}^{35}$ found an inverse relationship between MPOD (measured psychophysically by heterochromatic flicker photometry) and age, current or prior use of tobacco, and family history of AMD in a study of 828 healthy subjects from an Irish population. Beatty et $\mathrm{al}^{30}$ demonstrated an age-related decline in MPOD among healthy volunteers, and found significantly lower MPOD levels in healthy eyes predisposed to AMD (advanced forms of the disease were present in the contralateral eye). Kirby et a ${ }^{134}$ showed the presence of a central dip in the spatial profile of 
macular pigment in older subjects and in current cigarette smokers. The authors also found a typical exponential profile of macular pigment in younger subjects and the presence of a central dip in older individuals. The hypothesized common link between the risk factors of age and cigarette smoking is oxidative stress, which may prevent conversion of lutein into mesozeaxanthin in the macula. ${ }^{34}$ Our findings are consistent with this hypothesis, confirming decreased central MPOD values and a significant negative correlation with age, after a peak in early adulthood, in a normal population.

There are some notable limitations in this study. First, we included a relatively small population with a preponderance of female subjects. Second, although we excluded individuals using supplements known to enhance macular pigment, dietary surveys were not conducted. Third, it would have been helpful to have had a more homogenous distribution of patients according to age group. As described in the methods section, we consecutively enrolled healthy volunteers and, based on our exclusion criteria, those with any ocular diseases (including cataract), previous intraocular surgery, current consumption of oral carotenoids, presence of diabetes, and best-corrected visual acuity worse than 20/20 were not included. This fact clearly favored the inclusion of younger subjects during enrolment, given that most of these exclusion criteria, which are known to influence MPOD values (eg, diabetes) or to affect the quality of image acquisition (eg, cataract), are found in older subjects. Fourth, health status, eg, absence of diabetes, was established by questionnaire and not confirmed by laboratory test results. Finally, only patients with spherical equivalent $\pm 4 \mathrm{D}$ were included in our study, and the possible influence of refractive error on MPOD values was not evaluated. However, it is important to state that so far there is no evidence in the literature indicating the effect of refractive error on MPOD values. ${ }^{39,40}$ Despite these limitations, every effort was made to ensure that the subjects represented a normal ocular cohort, which would allow optimal images and data analysis.

In summary, this normal population of subjects tested with dual-wavelength autofluorescence demonstrated the characteristic distribution of MPOD, as previously described, with higher values in closest proximity to the foveal center. In addition, these levels appeared to increase during adulthood, followed by a gradual reduction after 60 years of age. If these findings can be confirmed in larger studies, we would advocate supplementation using lutein/zeaxanthin in older populations as prophylaxis against age-related maculopathy.
There is a need for further longitudinal studies to characterize better the effect of aging on MPOD.

\section{Disclosure}

The authors report no conflicts of interest in this work.

\section{References}

1. Sommerburg OG, Siems WG, Hurst JS, Lewis JW, Kliger DS, van Kuijk FJ. Lutein and zeaxanthin are associated with photoreceptors in the human retina. Curr Eye Res. 1999;19:491-495.

2. Nolan JM, Stack J, O'Connell E, Beatty S. The relationship between macular pigment optical density and its constituent carotenoids in diet and serum. Invest Ophthalmol Vis Sci. 2007;48:571-582.

3. Beatty S, Koh H, Phil M, Henson D, Boulton M. The role of oxidative stress in the pathogenesis of age-related macular degeneration. Surv Ophthalmol. 2000;45:115-134.

4. Bone RA, Landrum JT. Distribution of macular pigments components, zeaxanthin and lutein, in human retina. Methods Enzymol. 1992;213:360-366.

5. Rapp LM, Maple SS, Choi JH. Lutein and zeaxanthin concentrations in rod outer segment membranes from perifoveal and peripheral human retina. Invest Ophthalmol Vis Sci. 2000;41:1200-1209.

6. Trieschmann M, van Kuijk FJ, Alexander R, et al. Macular pigment in the human retina: histological evaluation of localization and distribution. Eye. 2008;22:132-137.

7. Bone RA, Landrum JT, Friedes LM, et al. Distribution of lutein and zeaxanthin stereoisomers in the human retina. Exp Eye Res. 1997;64:211-218.

8. Bone RA, Landrum JT, Hime GW, Cains A, Zamor J. Stereochemistry of the human macular carotenoids. Invest Ophthalmol Vis Sci. 1993;34:2033-2040

9. Landrum JT, Bone RA. Lutein, zeaxanthin, and the macular pigment. Arch Biochem Biophys. 2001;385:28-40.

10. Burstedt MS, Ristoff E, Larsson A, Wachtmeister L. Rod-cone dystrophy with maculopathy in genetic glutathione synthetase deficiency: a morphologic and electrophysiologic study. Ophthalmology. 2009;116:324-331.

11. Ding X, Patel M, Chan CC. Molecular pathology of age-related macular degeneration. Prog Retin Eye Res. 2009;28:1-18.

12. Madsen-Bouterse SA, Kowluru RA. Oxidative stress and diabetic retinopathy: pathophysiological mechanisms and treatment perspectives. Rev Endocr Metab Disord. 2008;9:315-327.

13. San Giovanni JP, Chew EY, Clemons TE, et al; Age-Related Eye Disease Study Research Group. The relationship of dietary carotenoid and vitamin $\mathrm{A}, \mathrm{E}$, and $\mathrm{C}$ intake with age-related macular degeneration in a case-control study: AREDS Report no 22. Arch Ophthalmol. 2007;125:1225-1232.

14. Nolan JM, Stack J, O’Donovan O, Loane E, Beatty S. Risk factors for age-related maculopathy are associated with a relative lack of macular pigment. Exp Eye Res. 2007;84:61-74.

15. Lima VC, Rosen R, Maia M, et al. Macular pigment optical density measured by dual wavelength autofluorescence imaging in diabetic and non-diabetic patients: a comparative study. Invest Ophthalmol Vis Sci. 2010;51:5840-5845.

16. Berendschot TT, van Norren D. Macular pigment shows ringlike structures. Invest Ophthalmol Vis Sci. 2006;47:709-714.

17. Delori FC, Goger DG, Keilhauer C, Salvetti P, Staurenghi G. Bimodal spatial distribution of macular pigment: evidence of a gender relationship. J Opt Soc Am A Opt Image Sci Vis. 2006;23:521-538.

18. Kirby ML, Galea M, Loane E, Stack J, Beatty S, Nolan JM. Foveal anatomic associations with the secondary peak and the slope of the macular pigment spatial profile. Invest Ophthalmol Vis Sci. 2009;50:1383-1391. 
19. Trieschmann M, Spital G, Lommatzsch A, et al. Macular pigment: quantitative analysis on autofluorescence images. Graefes Arch Clin Exp Ophthalmol. 2003;241:1006-1012.

20. Handelman GJ, Snodderly DM, Krinsky NI, Russett MD, Adler AJ. Biological control of primate macular pigment. Biochemical and densitometric studies. Invest Ophthalmol Vis Sci. 1991;32:257-267.

21. Wüstemeyer H, Moessner A, Jahn C, Wolf S. Macular pigment density in healthy subjects quantified with a modified confocal scanning laser ophthalmoscope. Graefes Arch Clin Exp Ophthalmol. 2003;241:647-651.

22. Canovas R, Lima VC, Garcia PM, Morini C, Prata TS, Rosen R. Comparison between macular pigment optical density measurements using two-wavelength autofluorescence and heterochromatic flicker photometry techniques. Invest Ophthalmol Vis Sci. 2010;51:3152-3156.

23. Delori FC, Goger DG, Hammond BR, Snodderly DM, Burns SA. Macular pigment density measured by autofluorescence spectrometry: comparison with reflectometry and heterochromatic flicker photometry. J Opt Soc Am A Opt Image Sci Vis. 2001;18:1212-1230.

24. Wustemeyer H, Jahn C, Nestler A, Barth T, Wolf S. A new instrument for the quantification of macular pigment density: first results in patients with AMD and healthy subjects. Graefes Arch Clin Exp Ophthalmol. 2002;240:666-671.

25. Delori FC. Autofluorescence method to measure macular pigment optical densities fluorometry and autofluorescence imaging. Arch Biochem Biophys. 2004;430:156-162.

26. Trieschmann M, Heimes B, Hense HW, Pauleikhoff D. Macular pigment density measurement in autofluorescence imaging: comparison of one-and two-wavelength methods. Graefe's Arch Clin Exp Ophthalmol. 2006;244:1565-1574.

27. Von Ruckmann A, Fitzke FW, Bird AC. Distribution of fundus autofluorescence with a scanning laser ophthalmoscope. Br J Ophthalmol. 1995;79:407-412.

28. Wooten BR, Hammond BR Jr. Spectral absorbance and spatial distribution of macular pigment using heterochromatic flicker photometry. Optom Vis Sci. 2005;82:378-386.

29. Richer S, Stiles W, Statkute L, et al. Double-masked, placebocontrolled, randomized trial of lutein and antioxidant supplementation in the intervention of atrophic age-related macular degeneration: the Veterans LAST study (Lutein Antioxidant Supplementation Trial). Optometry. 2004;75:216-230.
30. Beatty S, Murray IJ, Henson DB, Carden D, Koh HH, Boulton ME. Macular pigment and risk for age-related macular degeneration in subjects from a Northern European population. Invest Ophthalmol Vis Sci. 2001;42:439-446.

31. Ciulla TA, Hammond BR. Macular pigment density and aging, assessed in the normal elderly and those with cataracts and age related macular degeneration. Am J Ophthalmol. 2004;138:582-587.

32. Berendschot TT, van Norren D. On the age dependency of the macular pigment optical density. Exp Eye Res. 2005;81:602-609.

33. Bone RA, Landrum JT, Fernandez L, Tarsis SL. Analysis of the macular pigment by HPLC: retinal distribution and age study. Invest Ophthalmol Vis Sci. 1988;29:843-849.

34. Kirby ML, Beatty S, Loane E, et al. A central dip in the macular pigment spatial profile is associated with age and smoking. Invest Ophthalmol Vis Sci. 2010;51:6722-6728.

35. Nolan JM, Kenny R, O'Regan C, et al. Macular pigment optical density in an ageing Irish population: The Irish Longitudinal Study on Ageing. Ophthalmic Res. 2010;44:131-139.

36. Hammond BR Jr, Caruso-Avery M. Macular pigment optical density in a Southwestern sample. Invest Ophthalmol Vis Sci. 2000;41: 1492-1497.

37. Berendschot TT, Willemse-Assink JJ, Bastiaanse M, de Jong PT, van Norren D. Macular pigment and melanin in age-related maculopathy in a general population. Invest Ophthalmol Vis Sci. 2002;43:1928-1932.

38. Dietzel M, Zeimer M, Heimes B, Claes B, Pauleikhoff D, Hense HW. Determinants of macular pigment optical density and its relation to age-related maculopathy: results from the Muenster Aging and Retina Study (MARS). Invest Ophthalmol Vis Sci. 2011;52:3452-3457.

39. Neelam K, Nolan J, Loane E, et al. Macular pigment and ocular biometry. Vision Res. 2006;46:2149-2156.

40. Zheng W, Zhang Z, Jiang K, Zhu J, He G, Ke B. Macular pigment optical density and its relationship with refractive status and foveal thickness in Chinese school-aged children. Curr Eye Res. 2013;38:168-173.
Clinical Ophthalmology

\section{Publish your work in this journal}

Clinical Ophthalmology is an international, peer-reviewed journal covering all subspecialties within ophthalmology. Key topics include: Optometry; Visual science; Pharmacology and drug therapy in eye diseases; Basic Sciences; Primary and Secondary eye care; Patient Safety and Quality of Care Improvements. This journal is indexed on

\section{Dovepress}

PubMed Central and CAS, and is the official journal of The Society of Clinical Ophthalmology (SCO). The manuscript management system is completely online and includes a very quick and fair peer-review system, which is all easy to use. Visit http://www.dovepress.com/ testimonials.php to read real quotes from published authors. 